Online Appendix for the article

Flexible working for all? How collective constructions by Austrian employers and employees perpetuate gendered inequalities

\title{
Eva-Maria Schmidt
}

Published in: Journal of Family Research, https://doi.org/10.20377/jfr-668 
Table 1.

\begin{tabular}{|c|c|c|c|c|c|c|c|}
\hline Company & Sector & Employees & $\begin{array}{l}\text { Womens } \\
\text { quota, \% }\end{array}$ & $\begin{array}{l}\text { Part-time quota } \\
\text { women } / \text { men, \% }\end{array}$ & UF & Audit & Respondents \\
\hline C1_I & Trade & 36.800 & 75 & $46 / 7$ & $y$ & $y$ & ER1_I, EE3_I \\
\hline C2_I & Trade & 350 & $\sim 80$ & $57 / 13$ & $y$ & $y$ & ER2_I \\
\hline C3_I & Social/Health & 4.100 & 82 & $61 / 15$ & $y$ & $y$ & ER3_I \\
\hline C4_I & Infomation/ Media & 400 & 52 & $45 / 4$ & $n$ & $n$ & ER4_I, EE4_I, EE5_I \\
\hline C5_I & Trade & 420 & $\sim 30$ & $30 /-$ & $y$ & $y$ & ER5_I \\
\hline C6_I & Trade & 14 & 80 & each 100 & $y$ & $y$ & ER6_I \\
\hline C7_I & Construction & 134 & 35 & $21 / 4$ & $y$ & $y$ & ER7_I \\
\hline C8_I & $\begin{array}{l}\text { Information/ } \\
\text { Consulting }\end{array}$ & 150 & 55 & $49 / 4$ & $y$ & $y$ & ER8_I \\
\hline C9_I & Social/health & 8.282 & 79 & $62 / 16$ & $y$ & $\mathrm{n}$ & ER9_I \\
\hline C10_I & Real estate & 185 & 45 & $15 / 5$ & $y$ & $y$ & $\begin{array}{l}\text { ER10_I, EE1_I, EE2_I, } \\
\text { EE6_I }\end{array}$ \\
\hline C1_FG & Public & 6.260 & 66 & $43 / 12$ & $y$ & $y$ & $\begin{array}{l}\text { EE1_1/2/_FG, } \\
\text { ER1_FG }\end{array}$ \\
\hline C2_FG & Social/Health & 63 & 70 & $20-80$ & $y$ & $y$ & ER2_FG \\
\hline C3_FG & $\begin{array}{l}\text { Education/ } \\
\text { research }\end{array}$ & 493 & 66 & $46 / 36$ & $y$ & $y$ & $\begin{array}{l}\text { EE3_1/2_FG, } \\
\text { ER3_FG }\end{array}$ \\
\hline C4_FG & Industry & 1.236 & 29 & $7 / 3$ & $y$ & $y$ & ER4_1/2_FG \\
\hline C5_FG & Social/Health & $\sim 850$ & 70 & $45 / 31$ & $y$ & $y$ & ER5_FG \\
\hline C6_FG & Trade/service & 82 & 46 & $45 / 7$ & $y$ & $n$ & EE6_FG, ER6_FG \\
\hline C7_FG & Social/Health & $>1.000$ & $>75$ & - & $y$ & $n$ & EE7_FG \\
\hline C8_FG & Service & 1.118 & 61 & $42 / 9$ & $y$ & $y$ & ER8_FG \\
\hline C9_FG & Industry & 414 & 25 & $43 / 14$ & $y$ & $n$ & ER9_FG \\
\hline C10_FG & Traffic/ Transport & $\sim 40.000$ & 13 & - & $y$ & $n$ & $\begin{array}{l}\text { ER10_FG, } \\
\text { EE10_1/2/3/4_FG }\end{array}$ \\
\hline C11_FG & Tourism & 33 & 82 & $26 / 66$ & $n$ & $n$ & ER11_FG \\
\hline C12_FG & Tourism & 492 & 48 & $16 / 3$ & $n$ & $n$ & $\begin{array}{l}\text { ER12_1/2_FG, } \\
\text { EE12_1/2/3/4_FG }\end{array}$ \\
\hline C13_FG & Service/Media & 20 & 85 & $29 / 0$ & $y$ & $n$ & EE13_FG, ER13_FG \\
\hline C14_FG & Finance & 360 & 75 & $98 / 2$ & $y$ & $n$ & $\begin{array}{l}\text { EE14_1/2/3_FG, } \\
\text { ER14_FG }\end{array}$ \\
\hline C15_FG & $\begin{array}{l}\text { Information/ } \\
\text { Consulting }\end{array}$ & 627 & 36 & $23 / 7$ & $y$ & $n$ & ER15_FG \\
\hline C16_FG & $\begin{array}{l}\text { Finance/ } \\
\text { Insurance }\end{array}$ & 2.543 & 60 & $43 / 12$ & $y$ & $y$ & ER16_FG, EE16_FG \\
\hline C17_FG & Catering & 1.270 & 55 & $28 / 4$ & $y$ & $y$ & ER17_FG \\
\hline C18_FG & Trade & 7.516 & 68 & $86 / 14$ & $y$ & $y$ & ER18_FG \\
\hline C19_FG & Buildingindustry & 13.200 & 11 & $29 / 3$ & $y$ & $n$ & ER19_FG \\
\hline
\end{tabular}


Table 2.

\begin{tabular}{|c|c|c|c|c|c|}
\hline ID & Age & Gender & Education & Position & Other characteristics \\
\hline ER1_I & 38 & $f$ & University & Personnel development manager & Child(ren), Part-time (20h) \\
\hline ER2_I & 29 & $f$ & $\begin{array}{l}\text { Applied } \\
\text { university }\end{array}$ & Personnel manager & no children, Full-time \\
\hline ER3_I & 37 & $f$ & University & Personnel manager & Part-time (Parental part-time) \\
\hline ER4_I & 46 & $f$ & High school & Human resources manager & 1 child, Part-time (30h) \\
\hline ER5_I & 43 & $f$ & HAK & Personnel manager & Full-time \\
\hline ER6_I & 69 & $f$ & HBLA & Managing director & two children, Full-time \\
\hline ER7_I & 45 & $f$ & $\begin{array}{l}\text { Technical } \\
\text { Highschool }\end{array}$ & Managing director & two Children, Part-time (30h) \\
\hline ER8_I & 52 & $f$ & University & Personnel manager & \\
\hline ER9_I & 56 & $\mathrm{~m}$ & University Dr. & Personnel manager & Full-time \\
\hline ER10_I & 53 & $\mathrm{~m}$ & University & Personnel manager & \\
\hline ER4_1_FG & 48 & $f$ & University & Head of HR & \\
\hline ER4_2_FG & 53 & $f$ & Apprenticeship & Manager Payroll & \\
\hline ER5_FG & 54 & $\mathrm{~m}$ & University & Personnel manager & \\
\hline ER9_FG & 21 & $\mathrm{~m}$ & $\begin{array}{l}\text { Technical } \\
\text { Highschool }\end{array}$ & $\mathrm{HR}$ & \\
\hline ER10_FG & 53 & $f$ & University Dr. & $\begin{array}{l}\text { Personnel manager/Gender and } \\
\text { Diversity }\end{array}$ & \\
\hline ER12_1_FG & & $f$ & & HR Manager & \\
\hline ER12_2_FG & & $f$ & University & Director Sales & \\
\hline ER17_FG & 41 & $f$ & University BA & Personnel manager & \\
\hline ER1_FG & 64 & f & University & $\begin{array}{l}\text { Personnel manager/Gender and } \\
\text { Diversity }\end{array}$ & \\
\hline ER3_FG & 58 & $f$ & University & $\begin{array}{l}\text { Personnel manager/Gender and } \\
\text { Diversity }\end{array}$ & \\
\hline ER6_FG & 61 & $f$ & University & Managing director & Part-time \\
\hline ER8_FG & 53 & $f$ & University & HR Manager & \\
\hline ER11_FG & 30 & $f$ & High school & Managing director & Full-time, no children \\
\hline ER14_FG & 46 & $f$ & University & HR Manager & \\
\hline ER2_FG & 45 & $f$ & MAS & Manager Healthcare & \\
\hline ER13_FG & 47 & $f$ & University & Managing director & 1 child \\
\hline ER15_FG & 49 & $f$ & High school & HR Manager & \\
\hline ER16_FG & 44 & $f$ & University & Head of HR & Full-time, no children \\
\hline ER18_FG & 29 & $f$ & University & Personnel manager/Family & Full-time; 2 Children $(1,5 ; 4)$ \\
\hline ER19_FG & 34 & $f$ & University Dr. & Legal expert personnel & Full-time, 2 Children $(3,4)$ \\
\hline
\end{tabular}


Table 3.

\begin{tabular}{|c|c|c|c|c|c|c|c|}
\hline ID & Age & Gender & $\begin{array}{l}\text { Working } \\
\text { hrs/week }\end{array}$ & Working time model & Profession & $\begin{array}{l}\text { Net salary } \\
\text { in €uro }\end{array}$ & Child(ren) \\
\hline EE1_I & 56 & $\mathrm{~m}$ & 40 & Full-time, Flextime & Information manager & $>3000$ & 2 \\
\hline EE2_I & 41 & f & 4 & $\begin{array}{l}\text { Home-Office, Part-time } \\
\text { (10h) }\end{array}$ & Cultural manager & 446 & $3(7,5,1)$ \\
\hline EE3_I & 48 & $f$ & $40-50 h$ & $\begin{array}{l}\text { flexible working, home } \\
\text { office }\end{array}$ & Trainer & 3.000 & - \\
\hline EE4_I & 50 & $f$ & 38,5 & Full-time, Flextime & Manager & 3.600 & $2(16,18)$ \\
\hline EE5_I & 42 & $f$ & 30 & Part-time, Flextime & Personnel accounting & 2.600 & 2 \\
\hline EE6_I & 49 & f & $25-30$ & $\begin{array}{l}\text { Part-time, on weekends } \\
\text { also }\end{array}$ & Culture educator & 1.200 & $2(26,16)$ \\
\hline EE1_1_FG & 54 & $f$ & $37,5-45$ & & Personnel & 3.500.- & $1(17)$ \\
\hline EE3_1_FG & 53 & $f$ & $39-45$ & Flextime & Psychologist & 2.250.- & $2(26,28)$ \\
\hline EE6_FG & 37 & $f$ & $30-33$ & Home-Office & Marketing Manager & 4.500.- & $2(5,0)$ \\
\hline EE10_1_FG & 40 & $f$ & $20-25$ & Tele work & $\begin{array}{l}\text { Personnel } \\
\text { development }\end{array}$ & 1.500.- & $2(6,9)$ \\
\hline EE10_2_FG & 38 & $f$ & $38,5-45$ & Shift work & Chief dispatcher & 3.650.- & $2(11,14)$ \\
\hline EE12_1_FG & 29 & $f$ & $40-50$ & & Manager assistant & - & - \\
\hline EE14_1_FG & 50 & $f$ & 40 & & Trade employee & 2.100.- & $1(19)$ \\
\hline EE3_2_FG & 45 & $\mathrm{~m}$ & $39-50$ & Flextime & $\begin{array}{l}\text { Applied university } \\
\text { lector }\end{array}$ & 3.300.- & - \\
\hline EE10_3_FG & 45 & $\mathrm{~m}$ & $39-46$ & & HR employee & 3.600.- & $2(10,12)$ \\
\hline EE13_FG & 45 & $f$ & $18-20$ & Home office & PR employee & 1.900.- & $2(11,14)$ \\
\hline EE14_2_FG & 59 & $f$ & $40-50$ & & Office employee & 2.500.- & $2(31,42)$ \\
\hline EE1_2_FG & 51 & f & $32-32$ & Flextime & Employee & $2.000,-$ & $2(13,16)$ \\
\hline EE7_FG & 32 & f & $37-38$ & flexible working hours & $\begin{array}{l}\text { Assistant to } \\
\text { personnel manager }\end{array}$ & $2.200,-$ & - \\
\hline EE10_4_FG & 33 & $\mathrm{~m}$ & $38,5-42$ & Flextime & HR legal expert & $3.500,-$ & - \\
\hline EE12_2_FG & 24 & $f$ & $40-45$ & & HR Executive & $1.550,-$ & - \\
\hline EE12_3_FG & 25 & $f$ & $40-50$ & & Ass. to HR Director & $1.800,-$ & - \\
\hline EE12_4_FG & 35 & $f$ & $40-50$ & & Director of Events & $2.100,-$ & - \\
\hline EE14_3_FG & 51 & $f$ & $35-40$ & Flextime & IT project manager & $2.100,-$ & $2(19,16)$ \\
\hline EE16_FG & 37 & $f$ & $40-40$ & Home-Office, Flextime & $\begin{array}{l}\text { Commuication } \\
\text { employee }\end{array}$ & $3.600,-$ & $1(5)$ \\
\hline
\end{tabular}

